\title{
Desnutrición Materna y su Influencia sobre el Feto y el Recién Nacido
}

\author{
Dres. : Jaime A. Barrios Amaya ${ }^{*}$ y Vespaciano Zapata Gómez ** \\ Estudio químico de hierro sérico, proteínas, \\ albúminas y globulinas, somatometría.
}

\section{INTRODUCCION}

Estudios realizados en países desarrollados y subdesarrollados revelaron, que cuando la dieta materna es inapropiada se presentan con más frecuencia abortos, partos prematuros y mortinatos $(2,7,9)$.

Para diagnosticar el estado nutricional pueden emplearse los siguientes métodos, en conjunto o en forma individual:

- Observaciones clínicas

- Medidas corporales

- Análisis de laboratorio

* Jefe, Departamento de Obstetricia y Ginecología Facultad de Medicina, Universidad de Cartagena.

** Profesor Depto. Obst. Gin. Fac. Med. Univ. de Cartagena.

Este trabajo fue realizado en la Clínica de Maternidad "Rafael Calvo C", Departamento de Obstetricia y Ginecología, Universidad de Cartagena.

Presentado en el Cursillo "Terapéutica del Embarazo", XV Congreso Colombiano de Obstetricia y Ginecología, Bucaramanga, diciembre de 1983.

Auspiciado por PRENUP - Minsalud Colombia
En la valoración del estado nutricional, la historia de las costumbres alimentarias permite una interpretación más exacta de los hallazgos. Los métodos de laboratorio para estimar el estado nutricional son más exactos que los otros dos métodos. Por los signos clínicos es difícil valorar el estado de nutrición, ya que pueden ser poco aparentes, excepto si existen deficiencias graves. Las medidas corporales sólo representan una valoración de los estados nutricionales en el pasado, en tanto que los análisis de laboratorio dan un índice actual del estado de nutrición $(10,11)$.

El desarrollo y crecimiento fetal adecuados in utero dependen de un aporte uniforme de nutrientes de la madre al feto, ya que el déficit en el ingreso dietético, la absorción inadecuada de nutrientes a partir del intestino, el metabolismo anormal de proteínas, lípidos, carbohidratos y micronutrientes en el organismo materno, la insuficiencia de circulación placentaria y la utilización anormal de nutrientes por parte del feto pueden canducir a un notable deterioro del desarrollo fetal. Durante mucho tiempo se creyó que el feto, como un parásito, ten ía capacidad para obtener de la madre 
todas sus necesidades dietéticas con entera independencia del estado nutricional de la misma. Sin embargo, estudios efectuados durante las dos últimas décadas, han brindado suficiente información para demostrar que la nutrición materna inadecuada o deficiente durante el embarazo puede ser causa de desmedro intrauterino y de menoscabo en cuanto al desarrollo del cerebro fetal (8).

El hierro es uno de los elementos cuyo análisis en la sangre es de vital importancia, puesto que proporciona conocimientos acerca del estado nutricional de un individuo. El hierro es indispensable en los procesos oxidativos del organismo, ya que transporta oxígeno a las células. La mayor parte del hierro sangu íneo se localiza en los glóbulos rojos, y se halla en la hemoglobina (hematina-globina). La determinación de hierro en sangre se utiliza para medir la concentración de hemoglobina $(3,4,5,6)$.

Desde hace ya muchos años se reconoció la importancia de los suplementos de hierro en el embarazo y estudios más recientes han subrayado el papel de otros oligoelementos como zinc, cobre y manganeso. (8)

La placenta y el feto a término contienen 250 a $300 \mathrm{mg}$ de hierro, y además son requeridos unos $500 \mathrm{mg}$ de este metal para la expansión del volumen sanguíneo de la madre. El suplemento de hierro necesario para el feto, placenta y madre durante el embarazo se ha estimado en $750-800 \mathrm{mg}$. Como la dieta normal no contiene más que 1-2 mg por día, es indispensable administrar como suplemento 30 a $50 \mathrm{mg}$ de hierro diarios. Los niveles de hemoglobina caen normalmente an el tercer trimestre de la gestación, debido en parte al incremento en el volumen del plasma y en parte a las mayores necesidades del feto. (8)

En nuestro medio se ha medido hierro sérico en sangre fetal, sólo para comprobar los valores; también se ha determinado hierro total, hemoglobina $y$ hemato- crito en sangre materna y fetal, también para comprobar sus valores, datos éstos que no se pueden relacionar con el estado nutricional porque estos trabajos sólo apōrtan valores aislados. Los estudios que se han hecho al respecto en otros medios no corresponden a las condiciones ecológicas, socio-económicas y raciales de nuestro medio, factores éstos que como antes hemos anotado tienen una gran incidencia en el estado nutricional de un individuo.

\section{MATERIAL Y METODOS}

Se escogieron para el estudio 68 embarazadas desnutridas que tuvieran un $20 \%$ menos del peso corporal en relación con la talla, y 14 embarazadas clínicamente normales, consideradas como controles, con el fin de comparar y establecer diferencias. Las muestras se tomaron en la sala de partos de la Clínica de Maternidad "Rafael Calvo C". A las madres se les extrajo sangre venosa durante el trabajo de parto; la sangre fetal se obtuvo por punción de la vena del cordón en el momento del parto. A todas las embarazadas se les midió concentración de hierro sérico, proteínas totales, albúmina, globulinas, hemoglobina y hematocrito. Iguales medidas bioquímicas se hicieron en la sangre de la vena del cordón, después de pinzado y separado el recién nacido, en la porción distal o sea la unida a la placenta, antes que ésta se desprendiera.

Utilizamos el método colorimétrico para la determinación de hierro sérico sin desproteinización, el Método del Biuret para la determinación de proteínas totales, y electroforesis en papel acetato de celulosa (método discontinuo) para la determinación de prote ínas séricas fraccionadas. A todos los recién nacidos se les midió talla y peso.

\section{RESULTADOS}

La concentración de hierro sérico en sangre venosa de las embarazadas norma- 
les fue de $93.94 \mathrm{mcg} / \mathrm{dl}$, con un desvío standard de $\pm 8.07 \mathrm{mcg} / \mathrm{dl}$. En la sangre del cordón fue de $165.24 \mathrm{mcg} / \mathrm{dl}$, con un desvío standard de $\pm 38.24 \mathrm{mcg} / \mathrm{dl}$ (véase Tabla I).

Tabla I

CONCENTRACION DE HIERRO SERICO EN EMBARAZADAS NORMALES. SANGRE MATERNA Y DEL CORDON

\begin{tabular}{|ll}
\hline MADRE: & $\bar{X}=93,94 \mathrm{mcg} / \mathrm{dl}$. \\
& $S= \pm 8,07 \mathrm{mcg} / \mathrm{dl}$. \\
\hline FETO: & $\bar{X}=165,24 \mathrm{mcg} / \mathrm{dl}$. \\
& $S= \pm 38,24 \mathrm{mcg} / \mathrm{dl}$.
\end{tabular}

Figura 1

CONCENTRACION DE HIERRO SERICO EN SANGRE MATERNA

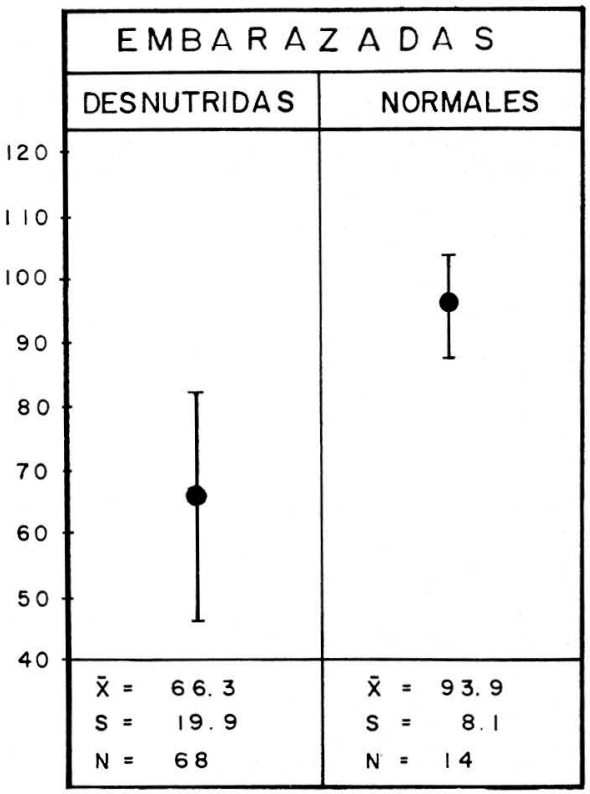

La concentración de hierro sérico en sangre venosa de las embarazadas desnutridas fue de $66.30 \mathrm{mcg} / \mathrm{dl}$, con un desvío standard de $\pm 19.86 \mathrm{mcg} / \mathrm{dl}$. En la sangre del cordón fue de $157.63 \mathrm{mcg}$ $/ d$, con un desvío standard de \pm 52.44 $\mathrm{mcg} / \mathrm{dl}$. (véase Tabla 2). La diferencia fue significativa. (Fig. 1).

\section{Tabla 2}

CONCENTRACION DE HIERRO SERICO EN EMBARAZADAS DESNUTRIDAS. SANGRE MATERNA Y DEL CORDON

\begin{tabular}{|c|c|}
\hline MADRE : & $\begin{array}{l}\bar{X}=66,30 \mathrm{mcg} / \mathrm{dl} \\
S= \pm 19,86 \mathrm{mcg} / \mathrm{dl}\end{array}$ \\
\hline FETO: & $\begin{array}{l}X=157,63 \mathrm{mcg} / \mathrm{dl} \\
S= \pm 52,44 \mathrm{mcg} / \mathrm{dl}\end{array}$ \\
\hline
\end{tabular}

Los valores de proteínas totales en embarazadas normales en promędio fueron de $6.61 \mathrm{~g} / \mathrm{dl}$, con un desvío standard de $\pm 0.53 \mathrm{~g} / \mathrm{dl}$. En la sangre del cordón el promedio fue de $6.07 \mathrm{~g} / \mathrm{dl}$, con un desvío standard de $\pm 0.55 \mathrm{~g} / \mathrm{dl}$ (véase Tabla 3 ).

Tabla 3

PROTEINAS TOTALES EMBARAZADAS NORMALES. SANGRE MATERNA Y DEL CORDON

\begin{tabular}{|lll|}
\hline MADRE : & $\bar{X}=6,6 \mathrm{l}$ & $\mathrm{g} / \mathrm{dl}$. \\
& $\mathrm{S}= \pm 0,53$ & $\mathrm{~g} / \mathrm{dl}$. \\
\hline FETO: & $\bar{X}=6,07$ & $\mathrm{~g} / \mathrm{dl}$. \\
& $\mathrm{S}= \pm 0,55 \mathrm{~g} / \mathrm{dl}$.
\end{tabular}


Los valores de proteínas totales en embarazadas desnutridas en promedio fueron de $6.69 \mathrm{~g} / \mathrm{dl}$, con un desvío standard de $\pm 0.8 \mathrm{~g} / \mathrm{dl}$. En la sangre del cordón el promedio fue de $5.84 \mathrm{~g} / \mathrm{dl}$, con un desvio standard de $\pm 0.88 \mathrm{~g} / \mathrm{dl}$. (véase Tabla 4 ).

\section{Tabla 4}

PROTEINAS TOTALES EN EMBARAZADAS DESNUTRIDAS. SANGRE MATERNA Y DEL CORDON

\begin{tabular}{|llll|}
\hline MADRE : & $\bar{x}=6,69$ & $\mathrm{~g} / \mathrm{dl}$. \\
& $\mathrm{s}= \pm 0,8$ & $\mathrm{~g} / \mathrm{dl}$. \\
\hline FETO & $\bar{X}=5,84$ & $\mathrm{~g} / \mathrm{dl}$. \\
& $\mathrm{S}$ & $= \pm 0,88$ & $\mathrm{~g} / \mathrm{dl}$.
\end{tabular}

Los recién nacidos de madres normales tuvieron una talla promedio de 48.69 $\mathrm{cm}$, con un desvío standard de \pm 1.49 $\mathrm{cm}$; un peso promedio de $3.316,1 \mathrm{~g}$, con un desvio standard de $\pm 392,9 \mathrm{~g}$ (véase Tabla 5).

Tabla 5

PESO $Y$ TALLA DE RECIEN NACIDOS DE MADRES NORMALES

\begin{tabular}{|c|c|c|c|}
\hline TALLA: & $\begin{array}{l}\bar{x} \\
s\end{array}$ & $\begin{array}{l}=48,69 \\
= \pm 1,49\end{array}$ & $\begin{array}{l}\mathrm{cm} . \\
\mathrm{cm} .\end{array}$ \\
\hline PESO: & $\begin{array}{l}\mathrm{X} \\
\mathrm{s}\end{array}$ & $\begin{aligned} & 3.316,1 \\
= & -392,9\end{aligned}$ & $\begin{array}{l}g \\
g\end{array}$ \\
\hline
\end{tabular}

Los recién nacidos de madres desnutridas tuvieron una talla promedio de 47.07 $\mathrm{cm}$, con un desvio standard de \pm 5.00 $\mathrm{cm}$; y un peso promedio de $3.041,4 \mathrm{~g}$, con un desvío standard de $\pm 523,4 \mathrm{~g}$ (véase Tabla 6).
Tabla 6

PESO Y TALLA DE RECIEN NACIDOS DE MADRES DESNUTRIDAS

\begin{tabular}{|c|c|c|c|}
\hline \multirow[t]{2}{*}{ TALLA } & $\bar{x}$ & $=47,07$ & $\mathrm{~cm}$. \\
\hline & $S$ & $= \pm 5,00$ & $\mathrm{~cm}$. \\
\hline \multirow[t]{2}{*}{ PESO: } & $\bar{x}$ & $=3.041,4$ & g. \\
\hline & $\mathrm{S}$ & $= \pm 523,4$ & $\mathrm{~g}$. \\
\hline
\end{tabular}

En la Tabla 7 se resumen los valores de hierro sérico, proteínas, albúmina y globulinas, en embarazadas normales $y$ en desnutridas, tanto en sangre venosa materna como en la sangre del cordón.

La concentración de hierro sérico en embarazadas desnutridas osciló entre 46 y $86 \mathrm{mcg} / \mathrm{dl}$, con una frecuencia de $44 \%$ entre 49 y $79 \mathrm{mcg} / \mathrm{dl}$. La concentración de hierro sérico en embarazadas normales osciló entre 86 y $102 \mathrm{mcg} / \mathrm{dl}$.

La concentración de hierro sérico en la sangre del cordón de madres desnutridas osciló entre 105 y $210 \mathrm{mcg} / \mathrm{dl}$, con 24 casos con concentraciones por debajo del mínimo valor normal, que fue de 127 $\mathrm{mcg} / \mathrm{dl}$.

Las primigestantes desnutridas, con una edad promedio de 19 años, tuvieron valores promedio de hierro sérico en la sangre del cordón, de $154 \mathrm{mcg} / \mathrm{dl}$ (véase Tabla 8).

Las desnutridas gravida II, con una edad promedio de 21 años, tuvieron valores promedio de hierro sérico en la sangre del cordón, de $167 \mathrm{mcg} / \mathrm{dl}$.

Las desnutridas gravida III, con una edad promedio de 24 años, tuvieron un valor promedio de hierro sérico en la sangre del cordón de $129 \mathrm{mcg} / \mathrm{dl}$.

Las desnutridas gravida $V$ (grandes multíparas), con una edad promedio de 
Tabla 7

VALORES DE HIERRO SERICO, PROTEINAS, ALBUMINAS Y GLOBULINAS EN EMBARAZADAS NORMALES Y DESNUTRIDAS

\begin{tabular}{|c|c|c|c|c|c|c|c|}
\hline & \multirow{2}{*}{$\begin{array}{l}\text { FE SERICO } \\
(\mathrm{mcg} / \mathrm{dl} .)\end{array}$} & \multirow{2}{*}{$\begin{array}{l}\text { P. TOTALES } \\
(g / d l)\end{array}$} & \multirow{2}{*}{$\begin{array}{c}\text { AL BUMINA } \\
(\%)\end{array}$} & \multicolumn{3}{|c|}{$G L O B \cup L I N A S$} & \multirow{2}{*}{$\frac{\%}{\text { GAMMA }}$} \\
\hline & & & & $A L F A_{1}$ & $\mathrm{ALFA}_{2}$ & BETA & \\
\hline $\begin{array}{l}\text { MADRE } \\
\text { CONTROL }\end{array}$ & $\begin{array}{l}\bar{x}=93,9 \\
s= \pm 8,1\end{array}$ & $\begin{array}{l}\bar{x}=6,6 \\
s= \pm 0,5\end{array}$ & $\begin{array}{l}\bar{X}=52,5 \\
s= \pm 5,9\end{array}$ & $\begin{array}{l}\bar{x}=5,3 \\
s .= \pm 2,0\end{array}$ & $\begin{array}{l}\bar{x}=10,9 \\
s= \pm 1,9\end{array}$ & $\begin{array}{l}\bar{x}=11,2 \\
s= \pm 26\end{array}$ & $\begin{array}{l}\bar{x}=18,7 \\
s= \pm 6,5\end{array}$ \\
\hline $\begin{array}{l}\text { MADRE } \\
\text { DESNUTRIDA }\end{array}$ & $\begin{array}{l}\bar{x}=66,3 \\
S= \pm 19,9\end{array}$ & $\begin{array}{l}\bar{x}=6,7 \\
s= \pm 0,6\end{array}$ & $\begin{array}{l}\bar{x}=51,4 \\
s= \pm 2,3\end{array}$ & $\begin{array}{l}\bar{x}^{\prime}=5,9 \\
s= \pm 2,3\end{array}$ & $\begin{array}{l}x=9,6 \\
s= \pm 2,9\end{array}$ & $\begin{array}{l}X=31,4 \\
S= \pm 3,5\end{array}$ & $\begin{array}{l}x=20,9 \\
s= \pm 4,7\end{array}$ \\
\hline $\begin{array}{l}\text { FETO } \\
\text { CONTROL }\end{array}$ & $\begin{array}{l}\bar{x}=165,2 \\
s= \pm 38,2\end{array}$ & $\begin{array}{l}\bar{x}=6,1 \\
s= \pm 0,5\end{array}$ & $\begin{array}{l}\bar{x}=59,7 \\
s= \pm 4,8\end{array}$ & $\begin{array}{l}\bar{x}=4,5 \\
s= \pm 2,5\end{array}$ & $\begin{array}{l}\bar{x}=6,6 \\
s= \pm 1,4\end{array}$ & $\begin{array}{l}\bar{x}=6,9 \\
s= \pm 2,9\end{array}$ & $\begin{array}{l}\bar{x}=22, \\
s= \pm 4,4\end{array}$ \\
\hline $\begin{array}{l}110 \\
\text { DESNUTRIDO }\end{array}$ & $\begin{array}{l}8=157,6 \\
S= \pm 52,4\end{array}$ & $\begin{array}{l}x=3,8 \\
s= \pm 0,9\end{array}$ & $\begin{array}{l}x=89,8 \\
S= \pm 7,4\end{array}$ & $\begin{array}{l}R=4,1 \\
S= \pm 1,5\end{array}$ & $\begin{array}{l}8=6,2 \\
s= \pm 1,9\end{array}$ & $\begin{array}{l}X=7,3 \\
s= \pm 3,5\end{array}$ & $\begin{array}{l}x=21,9 \\
s= \pm 5,4\end{array}$ \\
\hline
\end{tabular}

Tabla 8

\section{EMBARAZADAS DESNUTRIDAS}

\begin{tabular}{|l|l|l|}
\hline PARIDAD & EDAD PROMEDIO & $\begin{array}{l}\text { CONCENTRACION } \\
\text { HERRO SERICO } \\
\text { SANGRE FETAL }\end{array}$ \\
\hline PRIMIPARA & 19 AÑOS & $154 \mathrm{mco} / \mathrm{dl}$ \\
\hline PARA II & 21 AÑOS & $167 \mathrm{mco} / \mathrm{dl}$ \\
\hline PARA II & 24 AÑOS & $129 \mathrm{meg} / \mathrm{dl}$ \\
\hline GRAN MULTIPARA & 26 ANOS & $110 \mathrm{meg} / \mathrm{dl}$ \\
\hline
\end{tabular}

26 años, tuvieron valores promedio de hierro sérico en la sangre del cordón de $110 \mathrm{mcg} / \mathrm{dl}$.

\section{CONCLUSIONES}

La concentración de hierro sérico en embarazadas normales fue superior a aquélla en embarazadas desnutridas.

La concentración de hierro sérico en la sangre del cordón en embarazadas normales fue moderadamente superior a los valores obtenidos en el cordón de las embarazadas desnutridas.

La concentración de hierro sérico en la sangre del cordón de las embarazadas normales fue $12 / 3$ veces mayor que el valor materno, mientras que la concentración media de hierro sérico en la sangre del cordón de las embarazadas desnutridas fue $21 / 3$ veces mayor que el valor materno correspondiente. Esto nos indicaría que el feto extrae cantidades adecuadas de hierro de la circulación materna, sin importar los niveles de hierro de la madre.

No hubo diferencias significativas en los valores de proteínas totales, albúmina, y globulinas entre embarazadas normales y desnutridas, lo que corrobora que estos valores son poco representativos de la desnutrición, sobre todo si ésta no es severa.

En cuanto a talla y peso, se registrarun valores menores en recién nacidos de 
madres desnutridas, pero sin diferencia significativa estadísticamente.

Las primigestantes desnutridas, especialmente las menores de 20 años, tuvieron bajos valores de hierro sérico, tanto ellas como sus recién nacidos, lo que puede deberse a la mala alimentación y a estar aún en etapa de crecimiento, requiriendo cantidades adicionales de hierro en su dieta alimenticia.

Se observó que los valores de hierro sérico en los recién nacidos de madres desnutridas disminuyeron considerablemente cuando éstas fueron multíparas (grávida V), posiblemente debido a que sus reservas de hierro se van agotando y su alimentación deficiente no alcanza a suplir estas necesidades.

\section{RECOMENDACIONES}

1. Para una mejor evaluación del estado de desnutrición sería conveniente no sólo determinar los valores de hierro sérico, sino también otras variables: capacidad de fijación de hierro, cantidad de transferrina, concentración de vitamina $\mathrm{C}$, concentración de cobre, hemograma.

2. Además de la determinación de proteínas totales, debería también evaluarse calidad y cantidad de aminoácidos.

3. Orientar a las embarazadas sobre el balance de una dieta alimentaria adecuada para su estado.

4. Realizar una campaña para resaltar la importancia que tiene asistir a la consulta prenatal.

\section{SUMMARY}

Newborns of malnourished mothers are smaller and weigh less than those of normal mothers, although such differences are not statistically significant.
5. Desde el punto de vista nutricional, la dieta materna debe proporcionar los principios necesarios para: a) mantener en buena salud tanto a la embarazada como al feto; b) permitir una lactancia materna adecuada, y c) mantener a la madre en buena salud en el período entre un embarazo $y$ otro.

Finalmente se debe tener en cuenta que un estado nutricional adecuado desde la concepción hasta la adolescencia de la futura madre es también un factor determinante de gran importancia en el desarrollo intrauterino del niño.(1)

\section{RESUMEN}

Se estudiaron 68 embarazadas desnutridas y 14 normales como control. La talla y el peso de los recién nacidos hijos de madres desnutridas, fueron menores que los de las madres normales, sin significación estadística. La concentración de hierro sérico en las embarazadas desnutridas estuvo entre 46 y $86 \mathrm{mcg} / \mathrm{dl}$, y la de las embarazadas normales fue de 86 a $102 \mathrm{mcg} / \mathrm{dl}$. La concentración de hierro sérico en los fetos de madres desnutridas, en su gran mayoría, estuvo por debajo del valor normal mínimo, que fue de 127 $\mathrm{mcg} / \mathrm{dl}$. Tanto primigestantes jóvenes como multíparas desnutridas tuvieron valores de hierro bajos en sangre durante el trabajo de parto $y$ en la sangre del cordón del recién nacido.

Para una mejor evaluación de la desnutrición materna y del recién nacido, deberían determinarse además: capacidad de fijación de hierro, cantidad de transferrina, concentración de vitamina $\mathrm{C}$, concentración de cobre, cantidad y calidad de aminoácidos.

The concentration of blood iron in malnourished pregnant women was found to be between 46 and $86 \mathrm{mcg} / \mathrm{dl}$, and that of normal pregnant women was between 86 and $102 \mathrm{mcg} / \mathrm{dl}$. The concen- 
tration of blood iron in fetuses of malnourished mothers was, in most cases we studied, under the lower normal value, which is $127 \mathrm{mcg} / \mathrm{dl}$.

Both young primigravidae and Malnourished multiparae had low values of blood iron during labor and in the umbilical cord blood.

\section{BIBLIOGRAFIA}

1. ARROYAVE, G., LECHTIG, A. Nutrición materna en una sociedad de bajo nivel socioeconómico. Aspectos Perinatales del Parto Prematuro, Argentina, El Ateneo, 1a. ed., 1974.

2. COOPER, et al. Nutrición y Dieta. Méjico Interamericana, 15a. ed., 1970.

3. GEIGY. Datos del simposio clínico internacional sobre Ferropenia. Aroza, Suiza, 1969.

4. GUERCI, A.A. Método de análisis clínico y su interpretación. Argentina, El Ateneo, 3a. ed., 1969.

5. HARROW, B., MAZUR, A. Bioquímica Básica. Méjico Interamericana, 9a. ed., 1967.

6. HARPER, H.A. Manual de Química Fisiológica. Méjico Manual Moderna, 3a. ed., 1971.
The capacity of iron fixation, the quantity of transferrin, the concentration of vitamin $\mathrm{C}$, the concentration of copper, and the quantity and quality of amino acids should also be determined for a more detailed evaluation of maternal and newborn malnutrition.

7. Instituto de Bienestar Familiar. Dirección de Nutrición. La desnutrición proteica calórica infantil. Cartagena, 1979.

8. MOGHISSI, K.S. Nutrición materna durante el embarazo. Clin. Obstet. Ginec. Norteamericana. Ed. Interamericana, 1978.

9. Organización Panamericana de la Salud. Nutrición, Desarrollo y Comportamiento Social. Publicación Científica No. 269. Michigan Depto. de Desarrollo Humano. Univ. de Michigan, 1973.

10. PLATA, R.E. Pediatría Práctica. Medellín, Instituto Colombiano de Bienestar Familiar, 2a. ed., 1976.

11. WILSON, E.D., FISHER, K.H., FUCUA, M.E. Fisiología de la alimentación. Méjico Interamericana, 1a, ed., 1964. 